\title{
Jerarquía de la masculinidad y su representación audiovisual en la serie Parks and Recreation*
}

\author{
Miguel Sánchez Maldonado** \\ Brenda Azucena Muñoz Yáñez ${ }^{* * *}$ \\ Recibido: 2016-04-16. Enviado a pares: 2016-04-25 \\ Aprobado por pares: 2016-05-18. Aceptado: 2016-06-09 \\ DOI: 10.22395/angr.v15n29a3
}

\begin{abstract}
Resumen
En este artículo se aborda el concepto de género como constructo sociocultural determinante de una jerarquía que incluye y margina las personas a partir de su representación de actos masculinos o femeninos ideales para un grupo social. La diferenciación discursiva del género es el producto de varias tecnologías sociales, como los medios de comunicación, de discursos institucionales, epistemologías varias y de las acciones de la vida cotidiana. También se examina el concepto de masculinidad hegemónica y su representación en productos audiovisuales a través de un análisis semiótico de la representación de personajes masculinos en la serie Parks and Recreation (Daniels y Schur, 2009). El análisis ofrece ejemplos de la jerarquía masculina y los rasgos de las masculinidades hegemónica, subordinada, alternativa y marginal.

Palabras clave: Masculinidad hegemónica, medios de comunicación, estudios de género, representación.
\end{abstract}

\footnotetext{
- El artículo se enmarca dentro de una investigación más amplia sobre la comunicación online de la Responsabilidad Social Corporativa, que se llevó a cabo tomando como estudio de caso las empresas líderes del mercado minorista español.

* Es doctora en Ciencias de la Información por el IE Universidad de Segovia y licenciada en Comunicación por la Universidad Complutense de Madrid. Ha trabajado como profesora en Publicidad y Relaciones Públicas, Periodismo y Comunicación Audiovisual de la Universidad Europea Miguel de Cervantes de Valladolid y también profesora de Periodismo en la Universidad de Valladolid.

Ha publicado más de una treintena de artículos fruto de sus líneas de investigación centradas en el ámbito de la comunicación corporativa, comunicación publicitaria, nuevas formas de comunicación publicitaria y comunicación periodística (ambient marketing, redes sociales, estructura de contenidos en webs, prensa escrita y publicidad...), varios capítulos de libro y dos libros. gomeznietob@gmail.com

..* Es licenciada en Publicidad y Relaciones Públicas por la Universidad Europea Miguel de Cervantes (Valladolid).gomeznietob@hmca.uva.es
} 


\title{
Masculinity Hierarchy and Its Audio-Visual Representation in the series Parks and Recreation
}

\begin{abstract}
In this article, the concept of gender is tackled as a socio cultural construction, which determines a hierarchy that includes and put people on the margin from its representation of male and female acts, ideal for a social group. Gender discourse differentiation is the product of several social technologies such as communication media, institutional discourses, several epistemologies, and daily life actions. The concept of hegemonic masculinity and its representation in audio-visual products is examined through a semiotic analysis of male characters in the series Parks and Recreation (Daniels and Schur, 2009). The analysis offers examples of masculine hierarchy and features of hegemonic, subordinated, alternative, and marginal masculinities.
\end{abstract}

Key words: Hegemonic masculinity, communication media, gender studies, representation. 


\section{Introducción}

Desde los estudios de las masculinidades se ha discutido que, a través de las representaciones masculinas de la televisión, el cine y los deportes, se refuerzan varias creencias respecto a los hombres tales como que son invulnerables, violentos, tienen una sexualidad descontrolada, y ante todo deben realizar actos heroicos (Kimmel, 2011). Además de otras estructuras de socialización, los medios de comunicación presentan simbólicamente a través de la publicidad (Smith, 2007), de las películas (Spicer, 2007) y las noticias (Rasmusson, 2007) normas sociales sobre las que las audiencias construyen un imaginario de la constitución del ser masculino o femenino, dando lugar a procesos de reestructuración genérica acordes con el momento histórico en el que se producen los mensajes.

El género es una categoría de identidad que se refiere al comportamiento, situación y relaciones sociales asociadas con ser hombre o mujer. Aunque se ha abordado más desde el punto de vista de la feminidad que de la masculinidad, esta diferencia establece una condición de marginalidad para ambos sexos.

El género, o más bien, las masculinidades y feminidades, son configuraciones de prácticas que se construyen y transforman en el tiempo. Estas configuraciones de identidad condicionan el comportamiento, las relaciones económicas, sociales y de poder asociadas con ser hombre o mujer; al mismo tiempo que permiten el desempeño y la continuidad del sistema social, establecen una condición de marginalidad para ambos sexos. A diferencia del sexo, que tiene que ver más con la conformación biológica del cuerpo, la masculinidad y la feminidad surgen de las regulaciones culturales del comportamiento y, por lo tanto, el género es entendido en relación a "cómo son representados los hombres y las mujeres" (Barker, 2004, p. 73).

En algunas corrientes de la investigación sociológica, el sexo y el género son abordados como categorías diferentes, en las que el primero es determinado por la biología, y el segundo, formado culturalmente. El debate sobre la constitución del género tradicionalmente oscilaba entre dos posturas: el determinismo biológico, en la que el género es determinado por las características fisiológicas del cuerpo, considerando predominantemente ciertos órganos para la diferenciación; y la socialización diferenciada, según la cual, el aprendizaje social es categórico en la formación de modelos masculinos y femeninos.

No obstante, como apunta Kimmel (2011), las diferencias y desigualdades entre los géneros no pueden ser explicadas por ninguna de las dos formas. Primero porque el ser masculino o femenino no se encuentra naturalmente enraizado en las características de los cuerpos: las diferencias sexuales son insignificantes en relación con los argumentos de igualdad social (Barker, 2004). Segundo, porque la dominación no puede ser únicamente explicada por la socialización que las personas aprenden desde la infancia para asumir los roles típicos de hombres y mujeres; el cuerpo es necesario para articular los dispositivos de poder, mecanismos disciplinarios y normalización social (Foucault, 1976).

En los estudios de género existe una corriente con enfoque crítico, encabezada por Judith Butler, en la que la distinción entre sexo y género se desvanece, pues propone que el 
concepto de sexo es también un constructo sociocultural al no hallarse elementos sólidos de diferenciación biológica que concurran fuera de la repetición de discursos culturales normativos (Butler, 1990 a). Es decir, el género no puede ser separado del cuerpo; ser hombre o mujer es el punto de partida para su construcción social. A partir de las diferencias físicas, los individuos inventan, reciben y adaptan características psicológicas, sociales y económicas, lo que resulta en una configuración de prácticas sociales entendidas como acciones y comportamientos específicos. La familia, la escuela, los medios de comunicación y todo su entorno social les enseñan a los individuos, explícita e implícitamente, la forma en que deben pensar, sentir y actuar. A partir de esto se elaboran dinámicamente la masculinidad y la feminidad, y se constituyen en estructuras que, a la vez, interactúan con otras estructuras como la clase y la raza.

Para Connell (2005) el género es una práctica social que se refiere constantemente a los cuerpos y a lo que hacen los cuerpos; es, además, una estructura que establece una serie de procesos y jerarquías que regulan la vida social de hombres y mujeres, la forma en que se relacionarán entre ellos y con otras estructuras sociales. Kimmel (2011) coincide en que el género, además de establecer la diferencia entre hombres y mujeres, también dicta relaciones de poder y condiciones de desigualdad.

Desde la perspectiva de Connell (2005), al género lo constituyen tres partes esenciales: las relaciones de poder, las relaciones de producción, y la catexis — la última como componente sensorial y de apego emocional—; a estas podemos agregar el cuerpo como primer y último lugar de inscripción de la diferencia genérico-sexual (Torras, 2007).

Torras (2007) señala que estructuralmente existe una modelación sobre los cuerpos y sus actuaciones sociales, que los jerarquiza en función de un ideal para cada ámbito de la vida; por ejemplo, como instrumento de producción económica, como vehículo consumidor, como cuerpo disciplinado por la religión, el deporte o la moda. Bajo el control y vigilancia de las instituciones se establece una jerarquización naturalizada y normativa que hace legibles a los cuerpos reduciéndolos al sistema binario hombre-mujer.

A través de los actos corporales diferenciados por el género, tales como el trabajo, el deporte o el sexo, las personas se involucran con otras personas y con las instituciones sociales. Es en esa negociación reflexiva que hombres y mujeres se constituyen en sujetos y, a la vez, se reestructura el mundo social. Es en ese proceso que se crean relaciones de poder y se establece una jerarquía dominada por quienes actúan en conformidad con un ideal hegemónico masculino que subordina cualquier acto femenino; el patriarcado es producto de esta estructuración (Connell, 2005).

La diferenciación discursiva del género es el producto de varias tecnologías sociales (siguiendo el concepto de De Laurentis, 1987), como los medios de comunicación, y de discursos institucionales, epistemologías varias y prácticas críticas, además de prácticas de la vida cotidiana. El género es el conjunto de los efectos producidos en cuerpos, comportamientos y relaciones sociales, por el aprendizaje de conductas representadas en las narrativas maestras de la biología, la medicina, la filosofía y las artes, que muestran exageradamente una masculinidad hegemónica o una feminidad idealizada. 
Para Butler, el género como acción pública y acto performativo, no es una elección radical, ni un proyecto que refleja una elección meramente individual, pero tampoco está impuesto o inscrito sobre el individuo (1990 b, p. 307). Por género performativo, Butler (1990 b) se refiere a que actuamos en función de unas pautas sociales que nos exceden en tiempo y espacio, y determinan nuestros actos. Decir que el género es performativo es referirse a él como un conjunto de acciones y no de atributos preexistentes a la actuación de las personas; pero performar los actos de género es identificarse con los rasgos del ideal hegemónico del género dominante, ya sea por complicidad o por subordinación. A través de los distintos mecanismos de disciplina, el individuo es obligado a actuar en el lugar que se le ha asignado, y debe seguir y asumir los contenidos que se asocian al mismo. La actuación que podamos encarnar con respecto al género estará siempre signada por un sistema de recompensas y castigos.

Esta actualización del concepto de género da lugar a diferentes formas de ejercer la masculinidad, y por lo tanto existen diferentes masculinidades que se relacionan en una jerarquía encabezada por una masculinidad hegemónica, que no es practicada por la totalidad de los hombres. Un sector de ellos se diferencia de quienes se desempeñan como cómplices de la subordinación y marginalización, y se desenvuelve en concordancia con lo que es considerado como femenino (Connell, 2005; Connell, 2000; Connell y Messerschmidt, 2005).

Aunque existen diferentes formas de ejercer la masculinidad, la mayoría de los hombres se apropian de los ideales de la masculinidad hegemónica (Connell, 2005), que básicamente consisten en una oposición a cualquier alternativa que es identificada inmediatamente como no masculina; así, los comportamientos de riesgo, la fuerza física, el estoicismo, la dureza emocional, y el papel de proveedor económico son parte del discurso dominante de la masculinidad.

La masculinidad hegemónica es una posición predominante en la jerarquía de géneros, pero al mismo tiempo es un sistema e ideología que sirve para la reproducción de la dominación de los hombres; quienes participan como cómplices de esta masculinidad disfrutan de los beneficios materiales, físicos y simbólicos de la subordinación de la mujer y de otras formas genéricas alternativas, de otras clases sociales, religiones y etnias. Como ideología, la masculinidad hegemónica, a través de su representación simbólica, estructura la manera en que las personas entenderán y experimentarán el mundo sin importar su sexo.

Según Levy (2007), la ideología de la masculinidad hegemónica consta de cuatro dimensiones: la idealización de un solo tipo de masculinidad, o estereotipo regularizador; la jerarquización de los individuos a partir del género, además de otras categorías sociales; la subordinación de la mujer; y la subordinación de los comportamientos femeninos, a partir de la formación de estereotipos de la feminidad. Es mediante esta ideología que la masculinidad hegemónica, aunque solamente sea ejercida por una minoría estadística, tiene carácter normativo, representando el modo legítimo de ser hombre en una sociedad y momento determinado. 
Además de la masculinidad hegemónica y las masculinidades subordinadas, existen formas marginales, que son aquellas conformadas por otras estructuras sociales — como la etnia, la raza y la clase social — que limitan la reproducción de prácticas propias de cada grupo; mientras que las masculinidades subordinadas se derivan de la orientación sexual o lo que causa placer. Demetriou (2001) agrega otro elemento para el mantenimiento de la jerarquía masculina: un pragmatismo dialéctico, en el que la masculinidad hegemónica puede reestructurarse incorporando elementos de otras masculinidades para mantener el control.

Por lo anterior no debe pensarse que el predominio de los hombres sobre las mujeres se da por sí solo y se reproduce por hábito. La hegemonía requiere de la vigilancia de los hombres para mantener el statu quo, el descrédito de las mujeres y la incorporación de rasgos de otras masculinidades que actualicen aquellos que ya no sean vigentes (Connell y Messerschmidt, 2005; Demetriou, 2001). En esta relación de vigilancia y castigo a lo que se sale a la norma, los hombres subordinados al ideal hegemónico reproducen por su cuenta las coacciones del poder y son simultáneamente sometedores y sometidos, "se convierten en el principio de su propio sometimiento" (Foucault, 1975/2000, p. 187). Los hombres y las mujeres, consciente o inconscientemente, participan, a través de su práctica diaria, en la reproducción de la estructura hegemónica de la masculinidad. Como explicaría Giddens, los agentes reproducen las condiciones que hacen posible su acción, por lo que "las estructuras no existen independientemente del conocimiento que tienen los individuos de lo que hacen en su vida diaria" (1984, p. 26).

Por lo anterior se puede decir que el género masculino como ejercicio del poder exige características como el control, el orden, la consecución de objetivos y la racionalidad; como estructuras productivas se manifiesta en el capitalismo patriarcal, la subordinación de las mujeres y la marginalización de quienes no se desenvuelven dentro de la configuración de prácticas masculinas; y finalmente como catexis se manifiesta en la sanción de la expresión de afecto entre personas del mismo sexo, la supresión de emociones y la negación de necesidades.

\section{La representación de la masculinidad en el cine y la televisión}

Las representaciones cinematográficas hollywoodenses refuerzan estereotipos heterogéneos de la masculinidad que, de acuerdo a Cohan y Hark (1993), se podrían resumir en que los personajes hombres dominan y determinan la acción de los personajes femeninos; se muestran anhelantes de las mujeres, del placer sexual y de legitimar su masculinidad a través de la violencia. En géneros del cine como el western y el terror, por ejemplo, el hombre debe ser muy masculino, valiente y estoico; y, además, se exacerba la violencia. En los géneros bélicos, de acción y terror, también, el comportamiento del hombre se ve guiado por su necesidad de aniquilar a su oponente; $y$, además, es frecuente que el cuerpo masculino, principalmente del varón blanco, sea un vehículo de exhibición de musculatura, proeza física y rudeza tenaz. Incluso en el film noir, donde se representan personajes femeninos aparentemente emancipados, se puede observar que las figuras 
arquetípicas de la mujer, sea como redentora o como destructora, conforman su personalidad en relación con el dominio que tienen de su sexualidad (cf. Dusckworth, 2010). Es decir, que la representación de los personajes femeninos está circunscrita a los términos que se establecen como dominantes desde la masculinidad: la sexualidad y la violencia. Mientras que el hombre debe ser muy masculino, valiente y estoico, la mujer u ofrece su sexualidad a cambio de integrar al hombre alienado de la sociedad a través del matrimonio (mujer redentora), o bien despliega su sexualidad y amenaza la posición jerárquica de la masculinidad de forma violenta (mujer destructora o femme fatale). No obstante, es importante precisar que, a pesar de que el mito de la femme fatale pudo haber sido actualizado a través del fim noir (Bornay, 1995), dicha figura sufre en la actualidad la misma trivialización que la misma Bornay detectó para la última década del siglo XIX. Pareciera que la manera de lidiar con la sobreexposición de este tipo de personajes femeninos consiste en reducir su protagonismo al plano decorativo mientras que los personajes masculinos son los que actúan y ejercen poder en los escenarios, como más adelante se explica que sucede en la serie analizada.

Domínguez-Ruvalcaba (2007), encuentra que, en el cine mexicano, principalmente en el género de la comedia ranchera, se inventó una supremacía machista basada en el desafío y la seducción. La homosocialidad entre hombres solo se da con actos de arrogancia, brío, competencia y venganza, que les sirven a los personajes masculinos para demostrar su poder sobre el otro; mientras que la heterosocialidad gira en torno a la seducción para conquistar a las mujeres, y el sentimentalismo para recuperarlas.

De acuerdo con Feasey (2008) en las series de ficción los personajes masculinos muestran como características principales la valentía, la fuerza y la dominación; son los proveedores de la familia. Entre más heroica sea la profesión que desempeñen —-médico, policía, bombero, súper héroe- deberán sacrificar más su participación en las tareas domésticas, las responsabilidades familiares y la intimidad emocional, además de establecer límites más separados entre el ámbito privado y el público. Los personajes que ejercen masculinidades alternativas (gais, hippies, amos de casa) son representados, tanto en las ficciones como en los realities, menos definidos por el trabajo y más en relación con prácticas superficiales del cuidado de la apariencia, la moda y el consumo material. Esto también parece ser así para los numerosos programas de cocina, donde la presencia de chefs masculinos ha ido en aumento, y los hombres se muestran libres para disfrutar del ocio y capaces de conciliar el ámbito público con el espacio doméstico.

En el mismo tenor, Courtenay (2011) explica que la representación de la salud en los medios de comunicación está diferenciada genéricamente. Por ejemplo, en las películas estadounidenses, los personajes masculinos aparecen con mayor frecuencia fumando, ingiriendo bebidas alcohólicas o usando drogas. En la televisión, al menos un personaje bebe alcohol en el 60 \% de los programas de horario estelar, y dos terceras partes de estos personajes son hombres. Algunas investigaciones (Signorielli, 1993, Courtenay, 2000 c, Nettleton, 2009; Engels, Hermans, Van Baaren, Hollenstein y Bot, 2009) revelan un vínculo entre el consumo del alcohol, el consumo de drogas, la exposición a riesgos y 
la masculinidad en los diversos medios de comunicación, una asociación que se refuerza aún más en los eventos deportivos. Las conductas violentas o antisociales son utilizadas como recurso eficaz por los personajes masculinos para cumplir con sus objetivos sin ninguna consecuencia negativa física o social. Sin embargo, la representación de la práctica deportiva, por tener más personajes hombres que mujeres, es la excepción a estos comportamientos de riesgo y falta de cuidado presentados en los medios de comunicación. La cocina diaria, el cuidado doméstico y la buena nutrición se representan como actos femeninos: en la televisión, la mayoría de las mujeres son amas de casa, y cocineras, aunque en los últimos años programas como The Naked Chef han tratado de presentar la cocina doméstica como un espacio también masculino "through the use of new lad language and the depiction of a broader lifestyle image" (Feasey, 2008, p. 130). Cabe destacar que, en los programas de cocina profesional y la cocina exterior, los hombres siempre han sido representados como líderes de un sector productivo -industria restaurantera - o como cocineros hábiles capaces de sobrevivir y dominar a la naturaleza. Por otro lado, las mujeres y niñas son caracterizadas frecuentemente más delgadas que los hombres y los niños en televisión, películas y revistas (Signorielli, 1993; Courtenay, 2000).

\section{La jerarquía de la masculinidad en Parks and Recreation}

Para ejemplificar lo expuesto, se presenta un breve análisis semiótico de la construcción simbólica de la masculinidad a través de la representación de los personajes masculinos de la serie de televisión Parks and Recreation (Daniels y Schur, 2009). El análisis semiótico permite identificar las formas de producción y circulación de discursos sobre un fenómeno significante, como la performance de la masculinidad.

Parks and Recreation (2009) es una comedia que fue transmitida por primera vez en Estados Unidos el 9 de abril de 2009 por la cadena NBC. Los productores de la serie son los guionistas Gregg Daniels y Michael Schur que participaron en los 125 episodios y trabajaron previamente en programas de comedia como King of the Hill, The Office y Saturday Night Live. El 24 de abril de 2015 se transmitió el último episodio de su séptima temporada, con el que completó 125 episodios. El programa narra el trabajo del departamento de parques y recreación del pueblo ficticio de Pawnee, Indiana y la carrera política de Leslie Knope (Amy Pohler) a través del formato de falso documental. Si bien la serie tiene como protagonista a una mujer, predominan los personajes masculinos quienes a través de sus identidades ejemplifican la jerarquía de la masculinidad. Actualmente, la serie está disponible en Netflix y continuamente aparece entre los shows de comedia más vistos en esta plataforma (Barone, M. et al., 2016; Stella, 2016).

Para realizar el análisis, primero se organizaron los términos masculino y femenino en cuadrados semióticos (Greimas, 1993) con la finalidad de clasificar los semas nucleares de la representación de la masculinidad en cuatro oposiciones generales: poder, relaciones sociales, relación con el mundo y manifestación plástica del personaje (figura 1). Los semas nucleares son aquellas características del contenido analizado que son indispensables para que el objeto de estudio sea lo que es; son los elementos diferenciadores del signo. 
La oposición de los términos masculino y femenino produce una jerarquía encabezada por la masculinidad tradicional; por la operación de aserción el término masculino necesita de la existencia del término femenino para tener sentido. Los semas nucleares, debajo de los términos, por homologación, fueron considerados como constitutivos de la representación simbólica de la masculinidad o no masculinidad de los personajes. La masculinidad alternativa surge simbólicamente por la operación de negación y contradicción de los semas nucleares de la masculinidad hegemónica; sus semas no corresponden directamente con los precedentes de la feminidad, pero sí los implican. En cambio, la masculinidad subordinada aparece como consecuencia de la implicación de la masculinidad, pues sus semas nucleares contradicen a los de feminidad (figura 1).

Posteriormente, se examinaron los 112 episodios de la primera a la sexta temporada de la serie para identificar los elementos simbólicos en la caracterización de los personajes masculinos, y seleccionar fragmentos de diálogo en los que aparecieran figuras sémicas con diferenciadores genéricos (es decir, que expresaran la manera en que se relacionan los opuestos) y así poder ubicar a cada personaje en la jerarquía de masculinidad, que en la forma de un continuo tendría en un extremo la masculinidad hegemónica tradicional expuesta por el patriarca de la oficina Ron Swanson (Nick Offerman) y en el otro la masculinidad marginal personificada por el inadaptado Jean-Ralphio Saperstein (Ben Schwartz).

Figura 1: Cuadro semiótico del clasema género

\begin{tabular}{|c|c|c|}
\hline Masculino (Hegemónico) & $\ll$ & Femenino (Marginal) \\
\hline & Oposición de poder & \\
\hline Jefe & Oposición de relaciones sociales & Subordinado \\
\hline Superior & Oposición de relación con el mundo & Inferior \\
\hline Dominar & Oposición de comportamientos de & Obedecer \\
\hline Valentía & salud & Cobardía \\
\hline Homosociabilidad & Oposición estética & Heterosociabilidad \\
\hline Heterocentrismo & & Diversidad sexual \\
\hline Solitario & & Gregario \\
\hline Racional & & Emotivo \\
\hline Introversión & & Extraversión \\
\hline $\begin{array}{l}\text { Violencia para resolver pro- } \\
\text { blemas }\end{array}$ & & $\begin{array}{l}\text { Negociación para resolver pro- } \\
\text { blemas }\end{array}$ \\
\hline Estado natural de las cosas & & Intervención sobre las cosas \\
\hline Independencia económica & & Dependencia económica \\
\hline Inconsciencia de la salud & & Consciencia de la salud \\
\hline $\begin{array}{l}\text { Consumo predominante de } \\
\text { carne }\end{array}$ & & $\begin{array}{l}\text { Consumo predominante de } \\
\text { vegetales }\end{array}$ \\
\hline Abuso de alcohol & & Moderación con el alcohol \\
\hline
\end{tabular}




\begin{tabular}{|c|c|c|}
\hline Masculino (Hegemónico) & $\ll$ & Femenino (Marginal) \\
\hline Inconsciencia sobre la imagen & & Consciencia de la imagen \\
\hline $\begin{array}{l}\text { Estatura mayor a la de persona- } \\
\text { jes femeninos }\end{array}$ & & $\begin{array}{l}\text { Estatura igual o menor a la de } \\
\text { los personajes femeninos }\end{array}$ \\
\hline Vestuario monocromático & & Vestuario policromático \\
\hline Vello facial prominente & & Lampiño \\
\hline$\uparrow$ & $\swarrow \searrow$ & $\uparrow$ \\
\hline No femenino (Subordinado) & 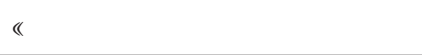 & No masculino (Alternativo) \\
\hline & Oposición de poder & \\
\hline No subordinado & Oposición de relaciones sociales & No jefe \\
\hline No inferior & Oposición de relación con el mundo & No superior \\
\hline No obedecer & Oposición de comportamientos de & No dominar \\
\hline No cobardía & salud & No valentía \\
\hline No heterosociabilidad & Oposición plástica & No homosociabilidad \\
\hline No diversidad sexual & & No heterocentrismo \\
\hline No gregario & & No solitario \\
\hline No emotivo & & No racional \\
\hline No extraversión & & No introversión \\
\hline $\begin{array}{l}\text { No negociación para resolver } \\
\text { problemas }\end{array}$ & & $\begin{array}{l}\text { No violencia para resolver pro- } \\
\text { blemas }\end{array}$ \\
\hline No intervención sobre las cosas & & No estado natural de las cosas \\
\hline No dependencia económica & & No independencia económica \\
\hline No consciencia de la salud & & No inconsciencia de la salud \\
\hline $\begin{array}{l}\text { No consumo predominante de } \\
\text { vegetales }\end{array}$ & & $\begin{array}{l}\text { No consumo predominante } \\
\text { de carne }\end{array}$ \\
\hline No moderación con el alcohol & & No abuso de alcohol \\
\hline No consciencia de la imagen & & No inconsciencia sobre la ima- \\
\hline No estatura igual o menor a la & & \\
\hline de los personajes femeninos & & No estatura mayor a la de per- \\
\hline No vestuario policromático & & sonajes femeninos \\
\hline & & No vestuario monocromático \\
\hline & & No vello facial prominente \\
\hline
\end{tabular}

Fuente: Elaboración propia

En los puntos intermedios estarían Chris Traegger (Rob Lowe), el administrador de la ciudad que rescata a Pawnee de la bancarrota, y representa una masculinidad hegemónica ascendente. Los hombres subordinados son dos: Ben Wyatt (Adam Scott), contador pú- 
blico con pocas habilidades sociales y pareja sentimental de Leslie Knope (Amy Pohler), y Jerry Gergich (Jim O'Heir), víctima de las bromas de los demás personajes. Más cercano al extremo de la marginalidad, está Tom Haverford (Aziz Ansari) quien ejerce una masculinidad alternativa; es el único personaje masculino con características étnicas distintas, es usuario ávido de la tecnología y seguidor de las últimas modas. Un caso especial es el de Andy Dwyer (Chris Pratt), pues representa una masculinidad hegemónica potencial; es el hombre infantil (manboy) que a pesar de sus 30 años todavía necesita de la ayuda del patriarca de la oficina y del administrador de la ciudad para actualizarse como hombre "de bien" a través del trabajo y del matrimonio.

En la tabla 1 se enlistan las características de los personajes y cuatro ejemplos lingüísticos para ilustrar la representación simbólica de cada tipo de masculinidad. Los dos hombres hegemónicos tienen en común que ejercen una posición burocráticamente más alta que la protagonista y el resto de los personajes; ambos son caucásicos, de ojos azules, independientes económicamente - Ron Swanson (Nick Offerman) trabaja en el Gobierno para desestabilizarlo desde el interior siguiendo una ideología libertaria y Chris Traeger (Rob Lowe) trabaja por placer para rescatar a gobiernos municipales en crisis-, se presentan como heterosexuales y galantes con las mujeres, son solitarios y en muy pocas ocasiones son representados en ámbitos privados. Sin embargo, mientras que Ron encarna a la masculinidad tradicional-.

Tabla 1: Jerarquización de los personajes, figuras sémicas, semas nucleares

\begin{tabular}{|c|c|c|c|}
\hline $\begin{array}{c}\text { Tipo de mascu- } \\
\text { linidad }\end{array}$ & Personaje & $\begin{array}{l}\text { Semas nucleares que } \\
\text { representa el personaje }\end{array}$ & Figuras sémicas en diálogos de la serie \\
\hline $\begin{array}{l}\text { Hegemónica } \\
\text { tradicional }\end{array}$ & $\begin{array}{l}\text { Ron } \\
\text { Swanson } \\
\text { (Nick } \\
\text { Offerman) } \\
\text { Caucásico, } \\
\text { ojos } \\
\text { azules, con } \\
\text { sobrepeso, } \\
\text { usa bigote. }\end{array}$ & $\begin{array}{l}\text { Jefe / Superior / Domi- } \\
\text { nar / Valentía / Homo- } \\
\text { sociabilidad / Hetero - } \\
\text { centrismo / Solitario / } \\
\text { Racional / Introversión } \\
\text { / Violencia para resol- } \\
\text { ver problemas / Estado } \\
\text { natural de las cosas / } \\
\text { Independencia eco- } \\
\text { nómica / Inconsciencia } \\
\text { de la salud / Consumo } \\
\text { predominante de carne } \\
\text { / Abuso de alcohol / } \\
\text { Inconsciencia sobre la } \\
\text { imagen / Estatura ma- } \\
\text { yor a la de personajes } \\
\text { femeninos / Vestuario } \\
\text { monocromático / Vello } \\
\text { facial prominente }\end{array}$ & $\begin{array}{l}\text { Poder: } \\
\text { "There are only three ways to motivate people: } \\
\text { money, fear, and hunger." } \\
\text { Relaciones sociales: } \\
\text { "Friends: one to three is sufficient." } \\
\text { Relación con el mundo: } \\
\text { "I also think it's pointless for a human to paint } \\
\text { scenes of nature when they can go outside and } \\
\text { stand in it." } \\
\text { Comportamientos de salud: } \\
\text { "Fish, for sport only, not for meat. Fish meat is } \\
\text { practically a vegetable." } \\
\text { Plástica: } \\
\text { "There are three acceptable haircuts: high and } \\
\text { tight, crew cut, buzz cut." }\end{array}$ \\
\hline
\end{tabular}




\begin{tabular}{|c|c|c|c|}
\hline $\begin{array}{l}\text { Tipo de mascu- } \\
\text { linidad }\end{array}$ & Personaje & $\begin{array}{l}\text { Semas nucleares que } \\
\text { representa el personaje }\end{array}$ & Figuras sémicas en diálogos de la serie \\
\hline $\begin{array}{l}\text { Hegemónica } \\
\text { emergente }\end{array}$ & $\begin{array}{l}\text { Chris } \\
\text { Traeger } \\
\text { (Rob Lowe) } \\
\text { Caucásico, } \\
\text { ojos azules } \\
\text { atlético, sin } \\
\text { vello facial. }\end{array}$ & $\begin{array}{l}\text { Jefe / Superior / Domi- } \\
\text { nar / Valentía / Hetero- } \\
\text { sociabilidad / Hetero- } \\
\text { centrismo / Solitario / } \\
\text { Emotivo / Extraversión } \\
\text { / Negociación para re- } \\
\text { solver problemas / In- } \\
\text { tervención sobre las } \\
\text { cosas / Independencia } \\
\text { económica / Conscien- } \\
\text { cia de la salud / Con- } \\
\text { sumo predominante } \\
\text { de vegetales / Mode- } \\
\text { ración con el alcohol / } \\
\text { Estatura mayor a la de } \\
\text { personajes femeninos } \\
\text { / Vestuario policromá- } \\
\text { tico / Lampiño }\end{array}$ & $\begin{array}{l}\text { Poder: } \\
\text { "As city manager, I play no favorites, but as } \\
\text { a private citizen, I'm free to support whomever } \\
\text { I choose. And I support Team Knope, because } \\
\text { they're the best! Everybody's the best. We're } \\
\text { all winners." } \\
\text { Relaciones sociales: } \\
\text { "I promised myself I was not going to cry tonight, } \\
\text { and I've already broken that promise five times. } \\
\text { But I will not break it a sixth." } \\
\text { Relación con el mundo: } \\
\text { "I bought my crib in Bloomington and I am } \\
\text { confident that it is literally the safest crib in the } \\
\text { explored universe." } \\
\text { Comportamientos de salud: } \\
\text { "I take care of my body above all else. Diet, exer- } \\
\text { cise, supplements, positive thinking. Scientists } \\
\text { believe that the first human being who will live } \\
150 \text { years has already been born. I believe I am } \\
\text { that human being." } \\
\text { Plástica: } \\
\text { "The world's my gymnasium, Ron!" }\end{array}$ \\
\hline Subo & $\begin{array}{l}\text { Ben Wyatt } \\
\text { (Adam Scott) } \\
\text { Caucásico, } \\
\text { ojos oscuros, } \\
\text { muy delgado, } \\
\text { sin vello fa- } \\
\text { cial. }\end{array}$ & $\begin{array}{l}\text { No subordinado / No } \\
\text { inferior / No obede- } \\
\text { cer / No cobardía / No } \\
\text { heterosociabilidad / } \\
\text { Heterocentrismo / No } \\
\text { gregario / Racional / } \\
\text { Introversión / Negocia- } \\
\text { ción para resolver pro- } \\
\text { blemas / Intervención } \\
\text { sobre las cosas / No } \\
\text { dependencia econó- } \\
\text { mica / No consciencia } \\
\text { de la salud / No mode- } \\
\text { ración con el alcohol / } \\
\text { No consciencia de la } \\
\text { imagen / No estatura } \\
\text { igual o menor a la de } \\
\text { los personajes femeni- } \\
\text { nos / No vestuario po- } \\
\text { licromático / Lampiño }\end{array}$ & $\begin{array}{l}\text { Poder: } \\
\text { "I'm not affraid of cops. I have no reason to be. } \\
\text { I never break any laws ever, because I'm deadly } \\
\text { afraid of cops." } \\
\text { Relaciones sociales: } \\
\text { "Nerd culture is mainstream now. So when you } \\
\text { use the word 'nerd' derogatorily, that means } \\
\text { you're the one that's out of the Zeitgeist." } \\
\text { Relación con el mundo: } \\
\text { "They are telling human stories in a fantasy } \\
\text { world." } \\
\text { Comportamientos de salud: } \\
\text { "Ben: I feel great, I ran } 5 k \text { this morning Leslie: } \\
\text { Really? } \\
\text { Ben: No, I threw up in the shower." } \\
\text { Plástica: } \\
\text { "I'll just put on my Star Wars pajamas and } \\
\text { sit in my mom's basement and pour over some } \\
\text { spreadsheets. It sounds great." }\end{array}$ \\
\hline
\end{tabular}


Jerarquía de la masculinidad y su representación audiovisual en la serie Parks and Recreation

\begin{tabular}{|c|c|c|c|}
\hline $\begin{array}{l}\text { Tipo de mascu- } \\
\text { linidad }\end{array}$ & Personaje & $\begin{array}{l}\text { Semas nucleares que } \\
\text { representa el personaje }\end{array}$ & Figuras sémicas en diálogos de la serie \\
\hline Alternativa & $\begin{array}{l}\text { Tom Haver- } \\
\text { ford } \\
\text { (Aziz Ansari) } \\
\text { Asiático } \\
\text { tamil, ojos } \\
\text { oscuros, } \\
\text { delgado, con } \\
\text { barba deli- } \\
\text { neada. }\end{array}$ & $\begin{array}{l}\text { No superior / No do - } \\
\text { minar / No valentía / } \\
\text { Heterosociabilidad / } \\
\text { Heterocentrismo / No } \\
\text { solitario / No racio - } \\
\text { nal / Extraversión / No } \\
\text { estado natural de las } \\
\text { cosas / No indepen- } \\
\text { dencia económica / } \\
\text { No inconsciencia de } \\
\text { la salud / No consumo } \\
\text { predominante de carne } \\
\text { / No abuso de alcohol } \\
\text { / Consciencia sobre la } \\
\text { imagen / Estatura igual } \\
\text { o menor a la de los } \\
\text { personajes femeninos } \\
\text { / Vestuario policromá- } \\
\text { tico / No vello facial } \\
\text { prominente }\end{array}$ & $\begin{array}{l}\text { Poder: } \\
\text { "Noo, Ron! My tummy's rumbling! It's scary... } \\
\text { Tommy's got the tum-rums." } \\
\text { Relaciones sociales: } \\
\text { "Warning! High levels of swagger coming } \\
\text { through! (Refereeing to himself)". } \\
\text { Relación con el mundo: } \\
\text { "Every day I start by hitting up Facebook, } \\
\text { Twitter, Tumblr and Instagram" } \\
\text { Comportamientos de salud: } \\
\text { "Ben: Should we throw in some salads for a } \\
\text { healthy option? } \\
\text { Tom: Wow, don't be such a Jerry, Ben." } \\
\text { Plástica: } \\
\text { "Oh, am I wearing an ascot? I didn't notice." }\end{array}$ \\
\hline Marginal & $\begin{array}{l}\text { Jean-Ral- } \\
\text { phio Sa- } \\
\text { perstein } \\
\text { (Ben } \\
\text { Schwartz) } \\
\text { Caucásico } \\
\text { italiano, ojos } \\
\text { oscuros, } \\
\text { muy delgado, } \\
\text { lampiño. }\end{array}$ & $\begin{array}{l}\text { Subordinado / Inferior } \\
\text { / Cobardía / Heteroso- } \\
\text { ciabilidad / Diversidad } \\
\text { sexual / Emotivo / Ex- } \\
\text { traversión / Interven- } \\
\text { ción sobre las cosa / } \\
\text { Dependencia econó - } \\
\text { mica / Consciencia de } \\
\text { la imagen / Estatura } \\
\text { igual o menor a la de } \\
\text { los personajes feme- } \\
\text { ninos / Vestuario po- } \\
\text { licromático / Lampiño }\end{array}$ & $\begin{array}{l}\text { Poder: } \\
\text { "Technically, I'm homeless" } \\
\text { Relaciones sociales: } \\
\text { "I sometimes call men beautiful too. I guess I'm } \\
\text { open-minded as hell." } \\
\text { Relación con el mundo: } \\
\text { "No, no, no, that's too much responsibility for } \\
\text { me". } \\
\text { Comportamientos de salud: } \\
\text { "I made my money the old fashioned way. Get- } \\
\text { ting run over by a Lexus" } \\
\text { Plástica: } \\
\text { "The bigger your hair, the bigger your glory." }\end{array}$ \\
\hline $\begin{array}{l}\text { Subordinada } \\
\text { madura }\end{array}$ & $\begin{array}{l}\text { Jerry Ger- } \\
\text { gich } \\
\text { (Jim O'Heir) } \\
\text { Caucásico, } \\
\text { ojos cafés, } \\
\text { obeso, lam- } \\
\text { piño. }\end{array}$ & $\begin{array}{l}\text { Subordinado / Inferior / } \\
\text { No obedecer / Valentía } \\
\text { / Heterosociabilidad } \\
\text { / Heterocentrismo / } \\
\text { Solitario / Emotivo / } \\
\text { No extraversión / Ne- } \\
\text { gociación para resolver } \\
\text { problemas / No inter } \\
\text { vención sobre las }\end{array}$ & $\begin{array}{l}\text { Poder: } \\
\text { "Chris: I believe you are capable of much more. } \\
\text { Jerry: I'm not." } \\
\text { Relaciones sociales: } \\
\text { "Donna: You'll never guess what I found on } \\
\text { Jerry's Facebook. } \\
\text { April: A friend? Buuurn." }\end{array}$ \\
\hline
\end{tabular}




\begin{tabular}{|c|c|c|c|}
\hline $\begin{array}{l}\text { Tipo de mascu- } \\
\text { linidad }\end{array}$ & Personaje & $\begin{array}{l}\text { Semas nucleares que } \\
\text { representa el personaje }\end{array}$ & Figuras sémicas en diálogos de la serie \\
\hline & & $\begin{array}{l}\text { cosas / Independencia } \\
\text { económica / No cons- } \\
\text { ciencia de la salud / } \\
\text { No consumo predo- } \\
\text { minante de vegetales / } \\
\text { Moderación con el al } \\
\text { cohol / No consciencia } \\
\text { de la imagen / Estatura } \\
\text { mayor a la de los per } \\
\text { sonajes femeninos / } \\
\text { Lampiño }\end{array}$ & $\begin{array}{l}\text { Plástica: } \\
\text { "Jerry: Tom, I really wish I could have your body. } \\
\text { April: Eww, like tied up naked in your basement? } \\
\text { Jerry: No, no I mean you're in good shape and } \\
\text { you can eat whatever you want. } \\
\text { Leslie: That was weird, Jerry." }\end{array}$ \\
\hline $\begin{array}{l}\text { No actuali - } \\
\text { zada }\end{array}$ & $\begin{array}{l}\text { Andy Dwyer } \\
\text { (Chris Pratt) } \\
\text { Caucásico, } \\
\text { ojos cafés, } \\
\text { obeso, con } \\
\text { barba descui- } \\
\text { dada. }\end{array}$ & $\begin{array}{l}\text { Subordinado / Inferior } \\
\text { / Obedecer / Valentía / } \\
\text { Homosociabilidad / } \\
\text { Heterocentrismo / Gre- } \\
\text { gario / No racional / No } \\
\text { introversión / No nego- } \\
\text { ciación para resolver } \\
\text { problemas / No estado } \\
\text { natural de las cosas / } \\
\text { Dependencia econó- } \\
\text { mica / Inconsciencia de } \\
\text { la salud / No consumo } \\
\text { predominante de vege- } \\
\text { tales / No moderación } \\
\text { con el alcohol / Incons } \\
\text { ciencia sobre la imagen } \\
\text { / Estatura mayor a la de } \\
\text { los personajes feme - } \\
\text { ninos / No vestuario } \\
\text { policromático / Vello } \\
\text { facial prominente }\end{array}$ & $\begin{array}{l}\text { Poder: } \\
\text { "By day. Andy Dwyer, shoeshinist. By different } \\
\text { time of day, Andy Radical, possum tackler. And } \\
\text { by night? Do whatever I want, no job." } \\
\text { Relaciones sociales: } \\
\text { "That is my band. I didn't recognize them without } \\
\text { me because I'm the only one that matters." } \\
\text { Relación con el mundo: } \\
\text { "Andy: I have no idea how to run a nonprofit. } \\
\text { April: Hey, you shined shoes for two years and } \\
\text { never earned a profit." } \\
\text { Comportamientos de salud: } \\
\text { "I once forgot to brush my teeth for five weeks." } \\
\text { Plástica: } \\
\text { "Can we by any chance stop at a place that has } \\
\text { free pants?" }\end{array}$ \\
\hline
\end{tabular}

Fuente: Elaboración propia.

Es completamente inexpresivo y distante, bebe güisqui en el trabajo, como pasatiempos practica la cacería, la carpintería, la construcción, consume carne y alimentos grasosos, y lleva bigote-, Chris representa un nuevo tipo de masculinidad hegemónica que ha adoptado rasgos de metrosexual — no toma alcohol, tiene una dieta vegetariana, depende de la tecnología, y es excesivamente expresivo. Aunque jerárquicamente Chris es el jefe de Ron, lo considera simbólicamente su superior.

La principal característica de los personajes subordinados, Ben Wyatt y Jerry Gergich, es que trabajan bajo las órdenes de Ron, Chris y de Leslie Knope. Cuando se les presentan problemas son negociadores, porque no tienen el cuerpo para demostrar su 
poder a través de la violencia física o el ingenio para usar violencia verbal. Ben, aunque es racional y rebelde, no tiene las competencias políticas ni sociales necesarias para ejercer un puesto de poder. Jerry es social y laboralmente incompetente. Ninguno de los personajes demuestra actos masculinos pertinaces, pero su hombría nunca es puesta en juicio basándose en su heterosexualidad, porque Ben es pareja de Leslie y Jerry está casado.

Tom Haverford personifica la masculinidad alternativa; del grupo de hombres es quien más convive con las mujeres de la oficina, el más consciente de la imagen personal y de la moda, utiliza colores llamativos y accesorios; es el que menos éxito tiene con las mujeres a pesar de ser galante; es débil físicamente y de menor estatura que los personajes femeninos. A pesar de estar casi en el límite de la jerarquía masculina, los hombres subordinados y los hegemónicos recurren a él para pedir consejos sobre moda, tecnología, relaciones humanas y expresión de los sentimientos, así dialécticamente se apropian de los rasgos que les ayudarán a mantenerse en el poder.

En el caso que hemos analizado, se puede advertir que la representación simbólica de la masculinidad a través de los personajes de Parks and Recreation (Daniels y Schur, 2009) contribuye a la aparente naturalización de ciertas prácticas culturales. La caricaturización de estos siete hombres aparenta ser inocente, pero se constituye como un nuevo mito del hombre masculino, del all-american guy. Según Barthes, los mitos son signos de segundo nivel que hacen posible el abuso ideológico oculto en la exposición decorativa que exige "que todos los hombres se reconozcan en esa imagen eterna y, sin embargo, situada en el tiempo que se formó de ellos en un momento dado como si debiera perdurar siempre" (1980, p. 137).

En las comedias de televisión no hay nada inocente; al contrario, se trata de los programas que más contribuyen a la estructuración de los ideales masculinos y femeninos; citando a Barker (2003) "donde hay signos, hay ideología". Sin embargo, es importante recalcar dos puntos respecto a este análisis que presentamos y la lectura del show por parte de la audiencia. En primer lugar, debemos tener en cuenta que el exceso semiótico en una serie como la que hemos analizado podría engendrar distintas significaciones en el proceso de descodificación del mensaje. Esto es importante sobre todo si se toma en consideración que los tonos de la comedia (sátira, ironía, parodia, absurdo) podrían ser elementales para guiar la lectura de la audiencia hacia una interpretación negociada. No obstante, hay que recordar que este exceso de significado trabaja siempre dentro de los límites establecidos por el productor del mensaje: a pesar de lo caricaturizado del personaje, Ron Swanson (Nick Offerman) siempre gana y ahí se están estableciendo ya los límites de la interpretación. En segundo lugar, también es importante recalcar que el hecho de que los productores de medios construyan guiones con representaciones de género hegemónicas no significa que los consumidores ejecutarán sus roles al pie de la letra; en el proceso de recepción existen múltiples formas de negociar los textos mediáticos que dan lugar a la variedad de identidades alternativas que existen en el mundo real como manifestación de la resistencia a la imposición estructural de la jerarquía masculina. 
Como colofón se podría mencionar que el éxito de la serie llevó a los productores a producir una séptima temporada situada en el año 2017 en el que se presenta una reconfiguración de la masculinidad de los personajes principales, cambios en las posiciones de la jerarquía de la masculinidad y el empoderamiento de los personajes femeninos. Esto podría parecer un posicionamiento político de los productores y una evidencia de la manera en que los programas de ficción contribuyen a la reestructuración de las normas y reglas del sistema social construyendo narrativas emergentes que se apropian de rasgos de los sectores alternativos de la población siguiendo el concepto de reestructuración de Giddens (1984).

\section{Referencias bibliográficas}

Barker, C. (2003). Cultural studies theory and practice. Londres: Sage.

Barker, C. (2004). The Sage dictionary of Cultural Studies. Londres: Sage.

Barthes, R. (1980). Mitologías. H. Schmucler (Trad.). México: Siglo veintiuno.

Bornay, E. (1995). Las hijad de Lilith. Madrid: Cátedra.

Butler, J. (1990 a). Gender Trouble. New York: Routledge.

Butler, J. (1990 b). Actos performativos y constitución del género: un ensayo sobre fenomenología y teoría feminista. En S. Case (Ed.), Performing Feminisms: Feminist Critical Theory and Theatre (pp. 270-282). Baltimore: Johns Hopkins University Press.

Cohan, S. y Hark, I. R (Eds.). (1993). Screening the male. Exploring masculinities in Hollywood cinema. Londres: Routledge.

Barone, M., Scarano, R., Aquino, T. y E. Sturges. (16 de marzo de 2016). The 50 Best TV Shows Streaming on Netflix Right Now. Recuperado de Complex: http://www.complex.com/pop-culture/ best-tv-shows-streaming-netflix/

Connell, R. (2000). Understanding Men: Gender Sociology and the New International Research on Masculinities. Clark Lecture, Department of Sociology, University of Kansas. Kansas City.

Connell, R. W. (2005). Masculinities (2 ${ }^{\text {nd }}$ Ed.). Berkeley, CA: University of California Press.

Connell, R. W. y Messerschmidt, J. W. (2005). Hegemonic Masculinity: Rethinking the Concept. Gender and Society, 19(6), 829-859.

Courtena, W. H. (2000). Engendering health: A social constructionist examination of men's health beliefs and behaviors. Psychology of Men and Masculinity, 1(1), 4-15.

Courtenay, W. H. (2011). Dying to be men. New York: Routledge.

Daniels, G. y Schur, M. (2009). Parks and Recreation [Serie de televisión]. New York: NBC.

De Lauretis, T. (1987). Techonologies of Gender. Essays on Theory, Film, and Fiction. Bloomington, IN: Indiana University Press. 
Demetriou, D. (2001). Connell's concept of hegemonic masculinity: a critique. Theory and Society, 30(3), 337-361.

Duckworth, A. R. (5 de agosto de 2010). Women in film noir I. The central archetypal roles. The Motley View. Journal of film, art and aesthetics. Recuperado de https://ardfilmjournal.wordpress.com/2010/08/05/ women-in-film-noir-i/

Strella, R. (4 de abril de 2016). Couch bound? Here are our favorite shows currently streaming on Netflix. Recuperado de Digital Trends: http://www.digitaltrends.com/movies/best-netflix-instant-tv-shows/

Domínguez-Ruvalcaba, H. (2007). Modernity and the nation in Mexican representations of masculinity. New York: Palgrave Macmillan.

Feasey, R. (2008). Masculinity and popular television. Edinburgo: Edinburgh University Press.

Foucault, M. (1975/2000). Vigilar y castigar (A. Garzón del Camino, Trad.). Buenos Aires: Siglo Veintiuno.

Foucault, M. (1976). The history of sexuality. Volume 1: An Introduction. (R. Hurley, Trad.). New York: Pantheon Books.

Giddens, A. (1984). The Constitution of Society. Berkeley: University of California Press.

Greimas, A. (1993). La semiótica del texto. Barcelona: Paidós.

Engels, R., Hermans, R., Van Baaren, R., Hollenstein, T., y Bot, S. (2009). Alcohol portrayal on television affects actual drinking behaviour. Alcohol and Alcoholism, 44(3), 244-249.

Kimmel, M. (2011). The gendered society $\left(4^{\text {th }}\right.$ Ed.). New York: Oxford University Press .

Levy, D. P. (2007). Hegemonic Masculinity. En M. Flood, J. Kegan Gardiner, B. Pease, y K. Pringle (Eds.), International Encyclopedia of Men and Masculinity (pp. 253-255). New York: Routledge.

Nettleton, P. (2009). Rescuing Men: The New Television Masculinity. (Tesis de doctorado, University of Minnesota). Recuperado de: http://conservancy.umn.edu/bitstream/handle/ 11299/57963/ Nettleton_umn_0130E_10769.pdf?sequence $=1$

Rasmusson, S. L. (2007). Journalism and the News. En M. Flood, J.K. Gardiner, B. Pease, K. Pringle (Eds.), International Encyclopedia of Men and Masculinity (pp. 351-354). New York: Routledge.

Signorielli, N. (1993). Mass media images and impact on health. Westport, CT: Greenwood Press.

Smith, T. (2007). Advertising. En M. Flood, J.K. Gardiner, B. Pease, K. Pringle (Eds.), International Encyclopedia of Men and Masculinity (pp. 3-5). New York: Routledge.

Spicer, A. (2007). Film. En M. Flood, J.K. Gardiner, B. Pease, K. Pringle (Eds.), International Encyclopedia of Men and Masculinity (pp. 212-213). New York: Routledge.

Torras, M. (2007). El delito del cuerpo. En M. Torras (Ed.), Cuerpo e identidad I (pp. 11-36). Barcelona: UAB. 
